\title{
Both multisensory stimulation and activity sessions improved mood and behaviour in dementia in the short term
}

\author{
Baker R, Bell S, Baker E, et al. A randomized controlled trial of the effects of multi-sensory stimulation (MSS) for people \\ with dementia. Br J Clin Psychol 2001 Mar;40:81-96.
}
QUESTIONS: In patients with dementia, is multisensory stimulation (MSS) more effective than activity sessions for improving behaviour and mood, and do the effects endure?

\section{Design}

Randomised \{allocation concealed*\}†, unblinded*, controlled trial with 1 month of follow up after 4 weeks of treatment.

Source of funding: not stated.

For correspondence

Ms J Holloway,

Department of

Psychological

Therapies, St Ann's

Court, Canford Cliffs,

Poole, BH13 7LN, UK

Fax +44 (0)120 270

6562.

Setting

3 day centres in Dorset, UK (home assessments were also done)

\section{Patients}

50 patients (mean age 78 y, $50 \%$ women) who lived at home with a primary caregiver; had a diagnosis of

Multisensory stimulation $v$ activity sessions for dementia

\begin{tabular}{lll}
\multirow{2}{*}{ Follow up } & \multicolumn{2}{l}{ Behaviour and Mood Disturbance mean score (SD) $\neq$} \\
\cline { 2 - 3 } & Multisensory stimulation & Activity sessions \\
\hline Baseline & $58.9(13.6)$ & $58.6(17.5)$ \\
\hline 4 sessions (mid treatment) & $54.2(12.7)$ & $59.5(22.0)$ \\
\hline 8 sessions (treatment end) & $54.1(12.7)$ & $61.2(23.0)$ \\
\hline 1 month after treatment & $59.4(14.1)$ & $59.0(21.9)$ \\
\hline
\end{tabular}

¥High score reflects worse outcome. Difference in change over time is statistically significant between groups.

\section{COMMENTARY}

MSS, or Snoezelen, facilities have become increasingly available in residential and daycare settings for people with moderate or severe dementia in the UK. Even in settings with limited resources, there has been interest in sensory stimulation through creative use of lights, music, aromas, and tactile objects. Client directed exploration of multisensory environments is concordant with the philosophy of person centred care, and common anecdotal reports from staff are that MSS enhances individual wellbeing. Previous studies on MSS for people with dementia, however, have been small and uncontrolled.

The exciting feature of this randomised controlled trial by Baker $e t a l$ is the evidence that MSS, delivered in a day centre, generalised to have a measurable effect on mood and behaviour in people with dementia in their own homes, as rated by a family caregiver. This generalisation effect was seen only for people in the MSS group and not for those receiving the control activity, although people in both groups were rated by staff as talking more spontaneously; relating better to others; doing more on their own initiative; and being more happy, active, and alert in the 10 minutes immediately after intervention sessions. Equally important is the observation of a negative withdrawal effect after the intervention stopped. Further work is necessary to generate data on optimal dose and duration of MSS.

Baker et al's findings complement the successes of cognitive stimulation provided by family members ${ }^{1}$ and by staff ${ }^{2}$ caring for people with mild to moderate dementia. They should provide the motivation for increased use of MSS procedures, with the goal of rehabilitation rather than palliation. This would, however, have implications for staff training and resources.

Georgina Charlesworth, MPhil, ClinPsyD University College London, London, UK

1 Quayhagen MP, Quayhagen M. Testing of a cognitive stimulation intervention for dementia caregiving dyads. In: Clare L, Woods RT, editors. Cognitive rehabilitation in dementia. Brighton, UK: Psychology Press, 2001.

2 Spector A, Orrell M, Davies S, et al. Can reality orientation be rehabilitated: development and piloting of an evidence-based programme of cognition-based therapies for people with dementia. In: Clare L, Woods RT, editors. Cognitive rehabilitation in dementia. Brighton, UK: Psychology Press, 2001.
Alzheimer's disease, or vascular or mixed dementia that was corroborated by the Cambridge Examination for Mental Disorders in the Elderly tool; had moderate to severe cognitive impairment (Mini-Mental State Examination [MMSE] score $0-17$ ); and attended 1 of 3 day centres $\geqslant 2$ days/week. Exclusion criteria were additional psychiatric diagnoses or $\geqslant 1$ MSS session in the previous 3 months. 2 patients dropped out of the MSS sessions, but all patients were assessed.

\section{Intervention}

Patients were allocated to 8 one to one sessions of MSS $(\mathrm{n}=25)$ or activity $(\mathrm{n}=25)$ for 4 weeks (two 30 minute sessions weekly). The MSS sessions involved stimulating all senses except taste; were non-directive and enabling; made no intellectual or physical demands on the patient; and used unpatterned, non-sequential stimuli. The activity sessions had specific aims and focuses (eg, jigsaw puzzles), were directive, used patterned and often sequential stimuli, and made intellectual and physical demands specific to the activity.

\section{Main outcome measures}

Behaviour, mood, and cognition were measured in homes and day centres using the Behaviour and Mood Disturbance (BMD) Scale, Hall and Baker's REHAB scale, and the MMSE. The immediate effect of sessions on behaviour and mood was also assessed (INTERACT SHORT scale).

\section{Main results}

Both groups showed immediate beneficial effects for 6 of 12 INTERACT SHORT items. After treatment, ratings of behaviour and mood at home improved more in the MSS group than the activity group (adjusted mean difference in change from baseline BMD score $-3.8,95 \%$ CI -7.6 to 0.0$)$. The activity group showed greater improvement than the MSS group in speech skill scores in day centres (adjusted mean difference in change from baseline REHAB score 0.8, CI 0.1 to 0.6). In the month after treatment ended, the MSS group had greater deterioration in BMD total score than the activity group, losing the improvements gained (table). Cognitive scores remained stable in both groups throughout the study.

\section{Conclusions}

In patients with dementia, both multisensory stimulation (MSS) and activity sessions had an immediate beneficial effect on behaviour and mood, which generalised to the home environment for MSS only. The effects were lost once treatment stopped.

*See glossary.

†Information provided by author. 